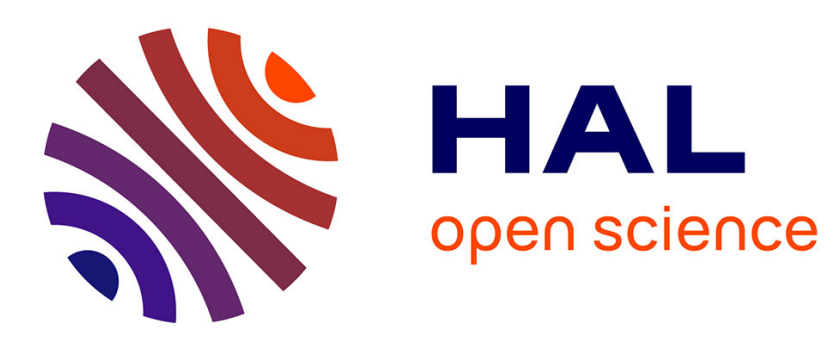

\title{
Oikiste et tyran : fondateur-monarque et monarque-fondateur dans l'Occident grec
}

Anne Jacquemin

\section{To cite this version:}

Anne Jacquemin. Oikiste et tyran: fondateur-monarque et monarque-fondateur dans l'Occident grec. KTÈMA Civilisations de l'Orient, de la Grèce et de Rome antiques, 1993, 18, pp.19-27. halshs00003913

\section{HAL Id: halshs-00003913 \\ https://shs.hal.science/halshs-00003913}

Submitted on 22 Apr 2005

HAL is a multi-disciplinary open access archive for the deposit and dissemination of scientific research documents, whether they are published or not. The documents may come from teaching and research institutions in France or abroad, or from public or private research centers.
L'archive ouverte pluridisciplinaire HAL, est destinée au dépôt et à la diffusion de documents scientifiques de niveau recherche, publiés ou non, émanant des établissements d'enseignement et de recherche français ou étrangers, des laboratoires publics ou privés. 


\section{Oikiste et tyran : fondateur-monarque et monarque-fondateur dans l'Occident grec}

L'Occident grec est associé à deux notions contradictoires, celle du monde nouveau des ámoıkíal, où chacun reçoit un lot égal, et celle du tyran encore au pouvoir quand ses pairs de Grèce propre ne sont plus qu'un souvenir. Il est vrai que ce monde égalitaire, dont on cherche les traces dans l'espace des villes et des campagnes $\left({ }^{1}\right)$, n'a guère duré $\left({ }^{2}\right)$ et l'anecdote du Corinthien imprévoyant qui troqua son lot de terre dans la future Syracuse contre un gâteau de miel $\left({ }^{3}\right)$ pourrait servir d'aition à l'existence de riches et de pauvres.

\section{L'OIKISTE PRIMUS INTER PARES OU MONARQUE}

L'expression de $\pi \rho \tilde{\omega} \tau o \varsigma \mu \varepsilon \tau \grave{a}$ ó $\mu$ í $\omega v$ qui rendrait l'idée d'une fonction de commandement exercée sur des égaux ne semble pas attestée et le terme d'íyź $\mu \omega v$ qui pourrait signifier cette notion s'est rapidement enrichi de l'idée de suprématie sur des inférieurs.

Les fondateurs des cités de Sicile sont des personnalités difficiles à saisir historiquement, puisque histoire et légende se mêlent souvent étroitement à leur propos. Les anecdotes retiennent surtout les défauts physiques qu'on leur attribue dans des traditions qui associent récits traditionnels et élaborations savantes nées dans des milieux liés à Delphes. Si la tare est une marque héroïque bien connue, que l'on songe à des figures comme Horatius Coclès ou Mucius Scaevola, les oikistes ont bien souvent ce caractère ; ainsi Myskellos, le fondateur de Crotone, est bossu, Battos, bègue, et Antiphèmos, incapable de rire. Dans ces deux derniers cas, on peut trouver l'origine de la particularité : le terme libyen «battos» qui désigne le roi $\left({ }^{4}\right)$, devenu un nom propre alternant avec Arkésilas dans la famille royale de Cyrène, a été rapproché du grec $\beta a \tau \tau a \rho i ́ \zeta \omega$, «bégayer», et a servi de base aux anecdotes de la consultation delphique pour trouver un remède et de la guérison due à la frayeur provoquée par un lion ${ }^{5}$ ); le nom

(1) Voir E. Lepore, "Problemi dell'organizzazione della chora coloniale», in: Problèmes de la terre en Grèce ancienne, sous la direction de M. I. Finley, Paris-La Haye, 1973, pp. 15-47 [= E. LeporE, Colonie greche dell'Occidente antico, Rome, 1989, pp. 79-110]: R. MARTIN, "Rapports entre les structures urbaines et les modes de division et d'exploitation du territoire", in: Problèmes de la terre en Grèce ancienne, pp. 97-112 [= R. MARTIN, Architecture et urbanisme, École française de Rome, 1987, pp. 582-597]; D. AdAmEsteanu, "Topografia e viabilità, in : Megale Hellas, sous la direction de G. Pugliese Carratelli, Milan, 1983, pp. 173-207; A. Di Vita Gafä, "L'urbanistica», in : Sikanie, sous la direction de G. Pugliese Carratelli, 2e éd., Milan, 1986, pp. 361-412.

(2) Voir E. Lepore, "Classi e ordini in Magna Grecia", in : Recherches sur les structures sociales dans l'Antiquité classique, Paris, 1970, pp. 43-62 [E. LePORE, op. cit., pp. 139-156].

(3) AthénêE, IV, 167d - citant Démétrios de Skepsis et Archiloque.

(4) Hérodote, IV, 155 : voir Fr. Chamoux, Cyrène sous la monarchie des Battiades, Paris, 1953, pp. 95-98.

(5) Hérodote, IV, 155; Pausanias, X, 15, 7. Cf. J. Defradas, Les thèmes de la propagande delphique, Paris, $1984^{2}$, pp. 245-254, et M. Giangiulio, «Deformità eroiche e tradizioni di fondazione - Batto, Miscello e l'oracolo delfico", ANSP, s. III, vol. XI.1, 1981, pp. 1-24. 
de la ville de Géla a été improprement rapproché du verbe $\gamma \varepsilon \lambda \dot{a} \omega$, «rire» (6), alors qu'il vient de celui du fleuve voisin, qui doit lui-même sa dénomination à la froideur de ses eaux ${ }^{7}$ ).

Rares sont les cas où la tradition nous renseigne précisément sur le milieu d'origine de l'oikiste. Un des exemples les mieux connus est celui d'Archias, le fondateur de Syracuse, membre de l'aristocratie des Bacchiades, ces "deux cents familles» corinthiennes $\left({ }^{8}\right)$, marginalisé par un meurtre involontaire $\left({ }^{9}\right)$. Vainement épris du jeune Actéon, fils d'un Argien bienfaiteur des Corinthiens, Archias avait tenté de l'enlever et le garçon était mort dans l'affrontement. Comme la position sociale du coupable l'empêchait d'obtenir justice, le père se tua dans le sanctuaire de l'Isthme, prenant à témoin Poseidon, qui exauça le malheureux en frappant la cité. Archias, qui avait été envoyé consulter Delphes, comprit le sens de l'oracle, ne rentra pas à Corinthe et partit fonder Syracuse, s'infligeant à lui-même l'exil. Cette anecdote qui associe les thèmes du meurtre involontaire, de la justice impossible à obtenir dans une oligarchie, quand on n'appartient pas au milieu dirigeant, ici une caste plus qu'une classe, du recours au dieu vengeur et de la justice immanente $\left({ }^{10}\right)$ a été jugée globalement véridique par A. E. Freeman. Ce dernier l'a rapprochée des rituels d'enlèvement crétois connus par Strabon ('11), alors qu'E. Will y a vu la projection sur une affaire de sang qui a pu être à l'origine de l'expulsion des Bacchiades d'un complexe mythico-rituel associant le chasseur Actéon, la figure de Mélissos-Mélicerte et des rites cathartiques et initiatiques pratiqués dans le sanctuaire de l'Isthme ( ${ }^{12}$ ).

Le chef de l'expédition comme les premiers pirates-marchands appartient à l'aristocratie (13), il a dès le départ un statut supérieur à celui de ses compagnons. Les fonctions qui sont les siennes témoignent de son importance, puisque c'est lui qui consulte l'oracle pour obtenir la sanction divine, qui commande l'expédition dont les navires sont fournis par l'État, comme il convient pour une entreprise politique $\left({ }^{14}\right)$. Il continue d'exercer ce commandement militaire, une fois la colonie fondée, si le besoin s'en fait sentir face à des indigènes qui refusent d'être spoliés de leurs terres. Il trace l'enceinte et établit la cité, même si on ne possède aucun témoignage ancien sur le rituel de fondation à l'époque archaïque ( $\left.{ }^{15}\right)$. Il apporte les images

(6) Voir Aristophane, Acharniens, 606.

(7) Voir Étienne de Byzance, s.v. $\Gamma \dot{\lambda} \lambda a$.

(8) Hérodote, V, 92. Cf. E. Wil.t, Korinthiaka, Paris, 1955, pp. 295-323.

(9) Diodore, ViII, 10 ; Plutarque, Amatoriae Narrationes, 2, 772c-773b; scholie à Apollonios de Rhodes, Argonautiques, IV, 1212. Ni Strabon (VI, 2, 4), ni Pausanias ne font allusion à ce drame.

(10) Dans le récit de Plutarque, l'exil ne suffit pas pour apaiser l'ombre du père, puisque Archias est tué par son mignon Tèléphos.

(11) A. E. Freeman, The History of Sicily from the earliest times, Oxford, 1891, I, p. 336sq., renvoyant à Strabon, $\mathrm{X}, 483$.

(12) E. WILL, Korinthiaka, pp. 180-187. Il paraît difficile de dater ce "bricolage» dont les Anciens étaient en partie conscients, puisque Diodore et Plutarque font expressément la comparaison entre le jeune Actéon et le chasseur trop curieux, qui connaissent le même sort: ils sont déchirés par ceux qui les aiment, amant et parents dans un cas, chiens dans l'autre. On notera cependant que le «nouveau fondateur» de Syracuse, le Corinthien Timoléon était également un meurtrier, puisqu'il avait tué son frère, qui aspirait à la tyrannie, et que son envoi était une sorte d'ordalie (DıODORE, XV1, 65, 4-9).

(13) Voir A. Mele, Il commercio greco arcaico - prexis ed emporie, Naples, 1979, pp. 58-78 («Il commercio prexis e il suo funzionamenton).

(14) Voir la façon dont Hérodote évoque la conduite singulière du Spartiate Dorieus (V, 42). Sur les fonctions de l'oikiste, voir M. CASEVITz, Le vocabulaire de la colonisation en grec ancien, Paris, 1985, pp. 101-107, et W. LESCHORN, "Gründer der Stadt» - Studien zu einem politisch-religiösen Phänomen der griechischen Geschichte, Stuttgart, 1984.

(15) Selon Aristophane, le premier rite est la dénomination de la nouvelle cité suivie d'un sacrifice aux dieux (Oiseaux, 809sq.). 
cultuelles de la métropole $\left({ }^{16}\right)$ et le feu du prytanée. Il nomme la nouvelle cité, la faisant ainsi exister ; comme le rappelle Platon ( ${ }^{17}$ ), la cité tire son nom de la topographie (lieu, rivière, source), d'une divinité ou d'un héros, voire de circonstances particulières. Platon ne mentionne pas le cas où la cité porterait le nom de son fondateur, à moins de ranger ce cas dans les circonstances particulières. Il existait pourtant au moins deux exemples dans le Pont-Euxin de cités qui portaient le nom de leurs fondateurs, Phanagoreia et Hermonassa, fondées au $\mathrm{VI}^{\mathrm{e}}$ siècle par deux bannis Phanagoras et Hermôn $\left({ }^{18}\right)$, mais il s'agissait d'expéditions montées par des individus, et non d'àđoıkíal voulues par des États.

Les fonctions exercées par l'oikiste supposent donc force et autorité $\left.{ }^{19}\right)$. Thucydide, dans sa présentation de la Sicile ${ }^{(20)}$, met d'ailleurs l'accent sur le rôle personnel de certains d'entre eux. Quand l'expédition est formée de contingents d'origines différentes, il doit pouvoir s'imposer pour créer l'unité $\left.{ }^{21}\right)$. Ceci est vrai également, lorsqu'il y a association avec des indigènes, comme à Léontinoi, même si l'élément indigène fut vite rejeté (22).

Au-dessus des colons, l'oikiste a bien des aspects d'un monarque, mais, si l'on excepte le cas de Battos à Cyrène $\left({ }^{23}\right)$, il n'est pas à l'origine d'une dynastie $\left({ }^{24}\right)$ : sa position particulière ne se traduit donc pas par le destin de sa famille, mais par son propre devenir posthume. Enterré au cœur de la cité qu'il a fondée, sur l'agora, il devient le protecteur de sa cité $\left({ }^{25}\right)$. Sa fête

(16) Voir la représentation de Tellis et de Kléoboia à la Leschè des Cnidiens à Delphes, œuvre du Thasien Polygnote : Pausanias, X, 28, 3 et J. Poullloux, Recherches sur les cultes et l'histoire de Thasos, I, Paris, 1954, pp. 25-27, 330.

(17) Lois, 704c.

(18) I. MALkin, «What's a Name? The Eponymous Founders of Greek Colonies», Athenaeum, N.S., 63, 1-2, 1985, p. 121 sq. On peut rapprocher de ces personnages le Crotoniate Philippe, qui, exilé, mène une trière qui lui appartient et dont il paie l'équipage (Hérodote, V,47).

(19) Au milieu du ve siècle, le décret athénien de fondation de Bréa $\left(I G, \mathrm{I}^{3}, 46\right)$ octroie les pleins pouvoirs à

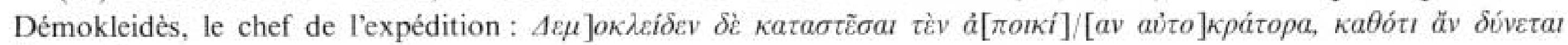
$\check{a}[\rho \imath \sigma \tau a]$.

(20) VI, 3-5.

(21) Quand l'expédition comprend des groupes venus de cités différentes, la tradition mentionne souvent deux, voire trois, oikistes, mais il semble qu'il y ait eu un oikiste principal et un oikiste secondaire en fonction de l'importance des contingents : ainsi, à Géla, le Crétois Entérios en retrait, presque oublié, alors que le Rhodien Antiphémos fait l'objet d'un culte. À Zancle, le débat sur le vrai fondateur paraît avoir été tranché par Delphes, ce qui serait à l'origine du rituel rapporté par Callimaque dans les Aitia, où les magistrats invitent l'oikiste au sacrifice sans le nommer : "qu'il vienne, favorable, à notre festin, celui qui édifia notre ville; qu'il en vienne deux et plus» (tr. G. VALLET).

(22) Voir POLyen, Stratagèmes, V, 5.

(23) Hérodote, IV, 159-162. Voir Fr. Chamoux, op. cit., pp. 128-210.

(24) Quelques rares rois sont attestés à l'époque archaïque dans des colonies d'Occident: si Aristophilidès, roi de Tarente vers 515 (Hérodote, III, 136), n'est sans doute qu'un magistrat qui porte ce titre (R. Drews, Basileus. The Evidence for Kingship in Geometric Greece, Yale, 1983, pp. 36-38; P. CARLIER, La royauté en Grèce avant

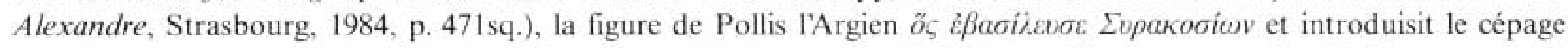
du biblinos thrace d'Italie en Sicile (Athenef, I, 3lab) est plus difficile à saisir : pour certains, Pollis est un vrai roi, que Syracuse ait connu une monarchie à l'origine - , mais on peut s'interroger sur la raison d'un Argien roi dans une colonie corinthienne, à moins que Syracuse n'ait été à l'origine une fondation argienne (R. VAN COMPERNOLLE, Kokalos, XII, 1966, pp. 75-101) - ou que la monarchie y ait succédé à une aristocratie originelle (E. A. FrEEMAN, op. cit., Oxford, 1891, II, pp. 8-10, 431-436), ce qui est peu vraisemblable ; pour d'autres, Pollis est une figure inventée par des érudits pour expliquer le nom porté par un certain cépage en Sicile (W. DunbАBın, The Western Greeks, Oxford, 1948, p. 93sq. ; R. Drews, op. cit., pp. 38-40) ; pour P. CARLier (op. cit., p. 467), si Pollis est un personnage de fiction, l'invention est plus ancienne que ne le prétend W. Dunbabin, puisque Athénée cite comme source l'historien Hippys de Rhégion de la première moitié du vo siècle.

(25) D. Asherı (La Sicilia antica, sous la direction d'E. GABbA et G. VAllet, Naples, 1980, I1, p. 111) émet cependant des réserves devant l'identification de la tombe de Thapsos aux coupes protocorinthiennes avec celle de 
est l'occasion de sacrifices héroïques et parfois de banquets, où les descendants du noyau originel réaffirment leur unité. À Géla on a découvert un pied de coupe attique du début du ve siècle avec une dédicace à Antiphémos de Rhodes, fondateur de la cité avec le Crétois Entérios (26). Lors des refondations de Timoléon au Ive siècle, le culte des oikistes connait un renouveau, qui est marqué notamment par la frappe de monnaies, comme les émissions de Géla au guerrier sacrifiant un bélier, en qui G. K. Jenkins $\left({ }^{27}\right)$ reconnait Antiphémos, ou les bronzes syracusains portant une tête barbue casquée, qui est à la fois Archias et Timoléon.

\section{LE TYRAN FONDATEUR DE CITÉS}

Certaines cités ont connu très tôt le phénomène de la tyrannie, comme Léontinoi avec Panaitios, qui gouverna peu de temps la cité à la fin du viI ${ }^{\mathrm{e}}$ siècle, ou Agrigente avec Phalaris, une des figures les plus noires de la légende du tyran avec son taureau d'airain $\left.{ }^{28}\right)$. Si la tradition attribue à ce dernier une politique d'expansion en territoire sicane et des visées sur la côte tyrrhénienne qui anticiperaient les plans de Thérôn, il n'est jamais présenté comme un fondateur. À la fin du vi ${ }^{e}$ siècle, le Spartiate Euryléôn, compagnon et successeur de Dorieus, présente une figure singulière d'oikiste remplaçant et infortuné à Hérakleia Minoa, de tyrannoctone - il débarrasse les Sélinontins de Peithagoras - et de tyran tué au pied de l'autel de Zeus Agoraios, mourant curieusement là même où on enterre les fondateurs $\left({ }^{29}\right)$. Ce tyrannoctone tenté par le pouvoir personnel est un personnage en avance sur son temps, un incompris qui aurait été plus à son aise cent cinquante ans plus tard $\left.{ }^{(30}\right)$.

Les tyrans de Sicile peuvent se ranger en deux catégories, les conquérants, qui ne visent qu'à accroître le territoire de leur patrie sans chercher à remodeler la cité, et les fondateurs, qui se veulent des sortes de démiurges. Ainsi Hippokratès de Géla n'a cherché qu’à s'étendre vers le Nord, sans avoir d'ailleurs des desseins aussi nets que ceux qu'a manifestés peu après Gélôn, même si les échecs qu'il a rencontrés notamment à la suite de la menace d'intervention de Corinthe et de Corcyre en faveur de Syracuse empêchent de prendre la juste mesure de ses projets ( $\left.{ }^{31}\right)$. Quand il s'est emparé de Kallipolis, de Naxos, de Zancle et de Léontinoi, il ne modifie pas la forme de ces cités. Le traitement de Camarine est également révélateur: devant l'avance des troupes gélôennes, les Syracusains ont fait évacuer leur colonie, dont ils livrent plus tard le territoire à Hippokratès pour payer la rançon de leurs prisonniers. La refondation de Camarine repeuplée de Gélôens n'a pas été voulue à l'origine par le tyran, mais c'est l'aristocratie syracusaine qui a détruit une nouvelle fois Camarine en intégrant sa population à celle de Syracuse. C'est là le signe d'une nouvelle politique : Syracuse a d'abord occupé le sud-est de la Sicile en fondant des colonies avant de concevoir une autre forme de domination de l'espace, celle de la grande cité.

l'oikiste malheureux Lamios. E. Greco attribue à Mégyllos ou Mégyllis, oikiste de Posidonia, l'hérôon à demi enterré de l'agora (Atti del ventisettesimo convegno di studi sulla Magna Grecia, Tarente 1987, 1988, pp. 485-486).

(26) M. Guarducci, Annuario, XXVII/IX, N.S. XI/XIII, 1949/51, p. 107, fig. 3 ; L. Dubois, Inscriptions grecques dialectales de la Sicile, École française de Rome, 1989, p. 159sq.

(27) The Coinage of Gela, Berlin, 1970, p. 114.

(28) Voir S. Bıanchett, Falaride e Pseudofalaride - Storia e leggenda, Rome, 1987.

(29) Hérodote, V, 46.

(30) Voir Kallippos, l'assassin et successeur de Diôn: Diodore, XVI,31,7 ; Plutarque, Dion, 57-58.

(31) Hérodote, VII, 154-155. 
La politique de Thérôn d'Agrigente ressemble à celle d'Hippokratès : son désir d'avoir un débouché sur la mer Tyrrhénienne a eu des conséquences importantes pour toute la Sicile, puisqu'elle a provoqué l'intervention carthaginoise, mais, devenu maître d'Himère, il s'est contenté d'y installer son fils Thrasydaios, qui s'est efforcé de mériter le nom de tyran au point que les Himériens ont fait appel à Hiérôn ( ${ }^{32}$ ). Anaxilas de Rhégion, que l'on peut considérer comme un tyran de Sicile à partir du moment où il règne également sur Zancle-Messana, a une politique plus audacieuse. Maître de Rhégion depuis 494, il s'empare de Zancle, tenue par des exilés samiens qui l'avaient ravie à Kadmos, fils et successeur de Skythès de Kos qu'Hippokratès avait placé à la tête de la cité. Il refonde Zancle avec une population d'origine variée comprenant des Messéniens de Grèce et donne à la cité le nom de Messana, qu'elle a gardée. Pendant plusieurs années, il gouverne les deux cités ensemble, avant de remettre Rhégion à son fils Léophrôn ( $\left.{ }^{33}\right)$. Si Anaxilas joue des groupes d'exilés - aristocrates samiens hostiles à la tyrannie qu'il envoie à Kalè Aktè, mais qui préfèrent la plus riche Zancle, Messéniens qui refusent le joug spartiate - il ne déplace pas des populations entières au gré de ses désirs.

Les vrais tyrans fondateurs sont les tyrans de Syracuse qui ambitionnent de régner sur la Sicile. «Tu règnes sur la Sicile» disent en 481 les envoyés des Grecs à Gélôn ${ }^{\left({ }^{34}\right)}$ et en 368 un décret

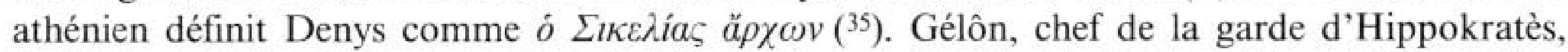
devient tyran de Géla en spoliant les fils de son maître, dont il reprend la politique. Plus ambitieux et plus heureux, il parvient à contrôler un vaste territoire, en sacrifiant, il est vrai, Géla dont la situation géographique est moins favorable que Syracuse. Devenu maitre de Syracuse à la faveur d'une guerre civile qu'il sut habilement exploiter ( $\left.{ }^{36}\right)$, il s'installa dans la cité, dont il devint véritablement le second fondateur, en la transformant en une $\mu \varepsilon \gamma a ́ \lambda \eta$

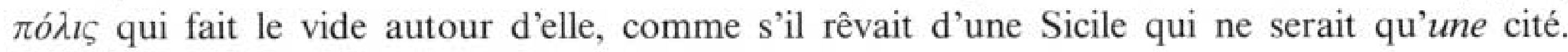
Dès l'abord, il sacrifie sa patrie, qu'il prive de la moitié de ses habitants avant de la remettre à son frère Hiérôn. Il installe des tyrans amis, comme Ainésidémos à Léontinoi ou le pugiliste Glaukos de Karystos à Camarine, politique qui rappelle celle d'Hippokratès. Après une révolte des Camariniens d'origine gélôenne, la cité est évacuée et ses citoyens deviennent des Syracusains. En revanche, lors des destructions de Mégara Hyblaea et de la petite cité d'Euboia, une colonie de Léontinoi, seuls les riches sont intégrés, les pauvres sont vendus comme esclaves à l'extérieur $\left({ }^{37}\right)$. La politique de Gélôn dans les années qui précèdent la bataille d'Himère vise à renforcer la puissance de Syracuse, qui devient la Cité de la Sicile orientale.

Quand Hiérôn succède à son frère en 478 , son seul désir est de surpasser son frère : l'éclat de ses participations aux concours d'Olympie et de Delphes, l'exploitation de sa victoire de Cumes manifestent une volonté tenace de faire mieux que Gélôn ( $\left.{ }^{38}\right)$. Fondateur, il l'est, et plus encore que Gélôn, puisqu'il crée une cité qu'il nomme. Profitant d'une éruption de l'Etna

(32) Diodore, XI, 48, 5-8.

(33) Hérodote, VI, 23 ; VII, 164.

(34) Hêrodote, VII, 157. Il faut certes tenir compte du désir de se rendre favorable Gélôn.

(35) $I G, \mathrm{II}^{2}, 103$ (M. N. Tod, A Selection of Greek Historical Inscriptions, p. 133). On retrouve la même formule dans le traité d'alliance conclu en $367: I G, \mathrm{II}^{2}, 105+523$ (M. N. ToD, op. cit., p. 136).

(36) Hérodote, VII, 155.

(37) Hérodote, VII, 156. Ironie tragique, la guerre avait été voulue par les riches de Mégare et le peuple s'y était d'abord opposé. L'historien ajoute que le tyran trouvait l'élément populaire fort incommode. Le rêve d'une grande cité se double de celui d'une cité aristocratique.

(38) Pindarf, Olympiques, I; Pythiques, 1 à III ; Pausanias, VI, 12, 1 (monument célébrant la victoire à la course de chars et à celle de chevaux montés lors du même concours olympique). 
qui détruit Naxos et Catane ( $\left.{ }^{39}\right)$, il fonde une nouvelle cité Aitna sur le territoire de Catane agrandi aux dépens des Sicules. Pour faire de cette cité dont il prend l'ethnique ${ }^{40}$ ) une $\mu \varepsilon \gamma a ́ \lambda \eta$ $\pi o ́ \lambda l \varsigma$, il fait venir les habitants de Catane, de Naxos, mais aussi ceux de Léontinoi, dont la ville n'avait pas souffert de l'éruption, et dix milie colons, venus pour moitié du Péloponnèse et pour moitié de Syracuse $\left({ }^{41}\right)$. L'originalité de la fondation d'Hiérôn tient à son statut plus qu'à l'importance de son territoire ou de sa population : alors qu'ailleurs le tyran coexiste avec une cité dont les institutions sont perverties, mais non détruites, Aitna est la propriété du tyran. Hiérôn place à la tête de cette cité, dont la naissance a été saluée par Pindare et par Eschyle, son fils Deinoménès encore enfant, à qui il donne pour tuteur Khromios ${ }^{42}$ ). Selon Diodore, il aurait fondé Etna par désir de recevoir les honneurs héroïques dus à un fondateur de cité et il les aurait effectivement obtenus $\left({ }^{43}\right)$.

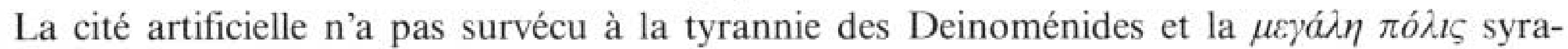
cusaine a connu des troubles à l'instauration de la démocratie, quand les citoyens de souche ont expulsé les mercenaires sicules qui devaient la citoyenneté aux tyrans $\left({ }^{44}\right)$. La destruction de la tombe d'Hiérôn à Aitna par les Catanéens qui recréent leur cité est un geste significatif : en expulsant l'oikiste, la cité se redéfinit. D’une certaine façon, les Deinoménides ont échoué dans leur projet de grande cité et la rivalité entre les deux frères a joué un rôle dans cet échec, mais le rêve d'une Syracuse splendide et isolée demeure vivant, alors même que les cités victimes de cette politique se reforment. Quel qu'en soit le désir, l'épisode tyrannique ne peut être effacé de l'histoire de la Sicile, car les mélanges de populations qu'il a suscités ont profondément transformé le faciès du pays : la part ionienne a perdu de son importance et l'inquiétude de cet élément explique les appels à Athènes, qui se veut la métropole de tous les Ioniens $\left({ }^{45}\right)$.

Au milieu du ve siècle, le Sicule Doukétios présente un cas singulier d'indigène tyran et oikiste. Plus qu'un nationaliste luttant contre les Grecs pour la liberté de ses frères, il est un émule des Deinoménides : il contribue à la refondation de Catane, mais offre un refuge aux Aitnéens expulsés qui étaient, il est vrai, pour nombre d'entre eux des Sicules; il fonde en 459 une

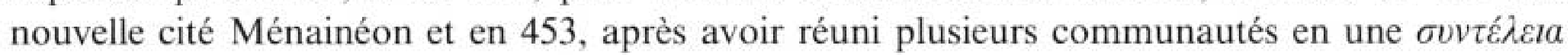

(39) Pindare qui se trouvait alors en Sicile pour l'exécution de l'ode olympique pour Hiérôn a donné une description de l'Etna en activité : Pythique, I, 19-28. Les Etnéennes d'Eschyle composées pour célébrer la fondation de la cité semblent associer mythe (les amours de Zeus et de Thalia, fille d'Héphaistos et la naissance des Paliques sortant de la terre après l'engloutissement de leur mère) et politique (accent mis sur la justice et sur l'œuvre d'Hiérôn) : cf. S. Radt, Tragicorum Graecorum Fragmenta, III, Göttingen, 1985, fr. 6, et Q. Cataudella, Atti del I. Congresso internazionale di studi sulla Sicilia antica, Kokalos, X-XI, 1964-1965, pp. 371-400. Les Paliques étaient des dieux

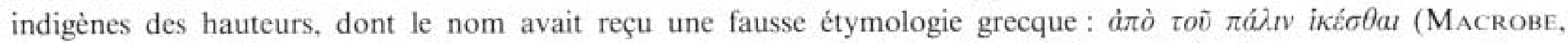
Saturnales, V, 19,17): voir L. BFIı,, «Ricerche sui Palici», Kokalos, VI, 1960, pp. 71-97. L'œuvre commandée au dramaturge athénien témoignait ainsi de la volonté du tyran de prendre en compte l'élément indigène, au prix certes de son hellénisation.

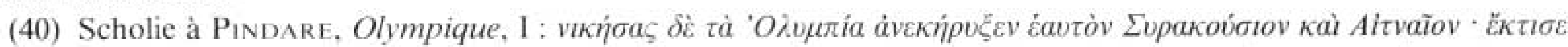

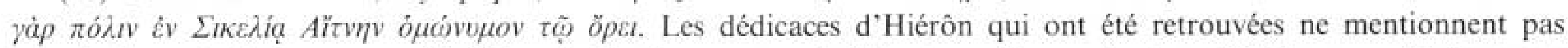
cet ethnique : le butin consacré à Olympie (trois casques ont été retrouvés) associent un Hiérôn sans ethnique aux Syracusains ; l'état de la dédicace du trépied de Delphes ne permet pas de se prononcer.

(41) Dionore, XI, 49, I. À la même époque, Thérôn fait venir des colons péloponnésiens pour repeupler Himère, dont il a massacré une partie des habitants (DıODORE, XI, 49, 3).

(42) Cet ami du tyran avait également une écurie de course et fut vainqueur à Némée et à Sicyone : PINDARF, Néméennes, I et IX.

(43) Diodore, X1, 49, 2 et $66,4$.

(44) Diodore, XI, 71, 3; 76, 4-6.

(45) Thucydide, III, 86,3; IV, 61, 4. 
(regroupement fiscal), la grande cité de Palikè, près du sanctuaire des dieux Paliques, au cœur du pays sicule oriental, pendant indigène de Syracuse ou d'Aitna $\left({ }^{46}\right)$. Le comportement de Doukétios est celui d'un tyran grec comme Gélôn ou Hiérôn : il octroie la citoyenneté à des mercenaires grecs ou sicules, il édifie un État puissant $\left({ }^{47}\right)$. La partie la plus singulière de sa carrière est cependant celle qui suit son échec : abandonné par les siens, il se rend aux Syracusains, qui l'exilent à Corinthe, d'où il revient en 448 à la tête de colons grecs pour refonder Kalè Aktè, dont l'oikiste est, après la mort de Doukétios, le chef sicule répondant au nom grec d'Arkhonidès $\left({ }^{48}\right)$.

Ces jeux de monarque ont tout brouillé et les distinctions ionien/dorien, grec/barbare ont perdu de leur pertinence. Le tyran, seul au-dessus d'une foule confuse, a dépassé ces clivages. On peut alors s'interroger sur le discours d'Hermocrate au congrès de Géla en $424\left({ }^{49}\right)$ : s'il s'agit assurément du discours d'un Syracusain plus que de celui d'un Sicéliôte, est-ce vraiment celui d'un démocrate? Comme le tyran de la première moitié du ve siècle est d'abord un chef de guerre, Hermocrate n'aurait-il pas imité les Deinoménides avant que les circonstances ne le transforme en héros de la résistance face aux Athéniens et aux Carthaginois ? Trop doué pour échapper aux regards soupçonneux de ses concitoyens, qui ne supportaient pas sa supériorité, il échoua en tentant de prendre par la force ce qu'on lui refusait. Ironiquement ce que le héros ne put accomplir, un «greffier» le fit. Denys reproduit Gélôn, même si une certaine tradition se plaît à opposer le bon souverain du ve siècle à l'abominable tyran du $\mathrm{I}^{\mathrm{e}}$ siècle : le discours de Théodôros en est un trop parfait exemple pour être crédible ( ${ }^{50}$ ).

En 405, face à l'offensive carthaginoise, Denys fait évacuer Géla et Camarine, alors que seule la première est assiégée, et la population de deux villes va grossir celle de Syracuse. Or les Agrigentins avaient quitté auparavant leur cité pour Géla $\left.{ }^{51}\right)$. Les Carthaginois jouent alors le rôle qu'avait tenu l'éruption de l'Etna sous Hiérôn. Lorsqu'il crée un groupe de «nouveaux citoyens» à partir de dépendants indigènes, Denys reprend la politique de la $\mu \varepsilon \gamma a ́ \lambda \eta$ $\pi o ́ \lambda l \varsigma$ créée en évacuant les cités alentour et en agrégeant des éléments hétérogènes, Grecs venus d'ailleurs ou barbares. En 404, il reprend Aitna, le refuge de ses opposants, Catane et Naxos, dont la population est vendue ( ${ }^{52}$ ). Catane, qui est donnée à des mercenaires campaniens, devient ainsi, après Entella, que les troupes à la solde du tyran avaient prise, le deuxième établissement italique en Sicile. Naxos est rasée et son territoire est donné aux Sicules, qui y fondent Tauroménion en 397 avec l'aide du Carthaginois Himilkon ( $\left.{ }^{53}\right)$. Quant à Léontinoi, elle est évacuée et ses habitants sont accueillis à Syracuse, qui devient avec la construction d'une vaste enceinte englobant les Épipoles la plus vaste cité enclose de Grèce : cité-armée au service d'un tyran qui détruit et créé les cités au gré des événements et de son caprice parfois. En 396 il refonde Messana détruite par les Carthaginois l'année précédente, en y installant des colons venus de Locres et de Medma, ainsi que des Messéniens chassés de Naupacte par les Lacédémoniens. Devant la protestation de Sparte, il déplace les six mille

(46) Diodore, XI, 76, 3; 78, 5;88, 6 .

(47) Voir D. Adamesteanu, «L'ellenizzazione della Sicilia ed il momento di Ducezio», Kokalos, VIII, 1962, pp. 167-198.

(48) Diodore, XI, 91-92; XII, 8.

(49) THUCYDIDE, IV, 59-64.

(50) Diodore, XIV, 65-69. Voir A. SCARpa Bonnzza Buora, Libertà e tirannide in un discorso "siracusano» di Diodoro Siculo, Rome, 1984, et J. Sanders, Dionysus I of Syracuse and Greek Tyranny, Londres, 1987, pp. 134-141.

(51) Diodore, XIII, 89.

(52) Diodore, XIV, 15.

(53) Diodore, XIV, 59. 
Messéniens et les utilise pour fonder Tyndaris en 395 à l'ouest de Mylai (54). Le projet italien du tyran dépassait de beaucoup les projets siciliens, mais il poursuivait d'une certaine façon la politique d'Hiérôn. Denys aurait voulu rattacher à la Sicile la partie méridionale de l'actuelle Calabre en la séparant du reste de l'Italie par un mur construit entre Skyllétion et Hipponion.

Les tyrans qui succèdent à Denys n'ont ni le temps ni les moyens d'une telle politique de bouleversements. Ils se contentent d'utiliser Syracuse comme champ d'expériences. La rôle de fondateur démiurge est en revanche assumé par Timoléon, qui libère la Sicile des Carthaginois et des tyrans. Même si la Sicile tend à se résumer à une cité, Syracuse, des efforts sont faits pour repeupler les cités. À la demande de Timoléon, les Corinthiens aux Isthmia et aux Pythia de 338 invitent les Grecs à émigrer en Sicile. L'archéologie a permis de retrouver des signes du repeuplement des cités, notamment à Agrigente et à Géla $\left.{ }^{55}\right)$. Cependant ce renouveau se fait au profit de Syracuse et au détriment des autres Grecs, des indigènes et des mercenaires campaniens engagés par les tyrans. Les rapports entre les nouveaux colons et les anciens habitants ne sont pas excellents. La retraite de Timoléon et sa mort ont sauvé le mythe du héros aimé de la Fortune ( $\left.{ }^{56}\right)$.

Si les Deinoménides ou Denys étaient des pseudo-rois, Agathocle, à la fin du ive siècle, prend le titre à l'imitation des diadoques $\left({ }^{57}\right)$. Sa politique qui vise à faire de Syracuse la Cité de Sicile lui aliène les aristocraties des autres cités, qui font appel aux Carthaginois. Plus audacieux que Denys, ce roi, qui reprend la politique des tyrans syracusains en Sicile, mais aussi en Italie méridionale, porte la guerre en Afrique. Malgré ses talents militaires et politiques, il ne peut fonder une dynastie de sorte que son royaume ne lui survit pas et que sa mort, comme presque toutes les disparitions de tyrans en Sicile, est suivie des troubles dus à la présence de mercenaires dans des cités qui retrouvent la liberté. Les Syracusains installent au port de Messana un groupe de Campaniens, les Mamertins, qui s'emparent de la cité et lancent des attaques contre Camarine et Géla, qu'ils détruisent ( $\left.{ }^{58}\right)$. Le tyran Phintias d'Agrigente transfère les habitants de Géla dans une nouvelle cité qu'il fonde à l'ouest et à laquelle il donne son nom $\left({ }^{59}\right)$; un tyran sans éclat est ainsi fondateur et éponyme d'une cité, ce que n'avaient point fait les Deinoménides, Denys ou Agathocle. En 284, Phintias imite Alexandre et les diadoques.

Fonder une cité, c'est faire acte de créateur. Tentation pour un tyran que de remodeler un territoire, acte difficile à accomplir par les tyrans de Grèce et d'Asie, à l'exception de Miltiade en Chersonnèse et des tyrans éponymes du Pont, mais entreprise réalisable dans cette terre

(54) Diodore, XIV, 78, 5-6.

(55) Voir entre autres P. ORLANDINI, «Storia e topografia di Gela del 405 al 282 a.C. alla luce delle nuove scoperte archeologiche», Kokalos, II, 1956, pp. 158-176; D. Adamesteanu, "La Rinascita della Sicilia nell'età di Timoleonte alle luce delle nuove scoperte archeologichen, Kokalos, IV, 1958, pp. 24-68, et les articles consacrés à Hérakleia Minoa, Camarine, Mégara Hyblaea, Morgantina, Tyndaris et à l'Ouest de la Sicile dans ce dernier volume de la revue. L'ouvrage de R. J. A TAL.BerT, Timoleon and the revival of Greek Sicily 344-317 B.C., Cambridge, 1974, met l'accent sur la confirmation apportée par l'archéologie à ce que les sources anciennes disent du renouveau de l'ile.

(56) Plutarque, Timoléon, 37-38. M. Sordi, qui rappelle la perspective panhellénique et le projet philosophique de l'œuvre de Timoléon, s'interroge sur ce que son action a apporté aux anciens habitants de la Sicile (La Sicilia dal 368/7 al 337/6, Milan, 1983, pp. 78-80).

(57) DiOdore, XX, 54, 1.

(58) Polybe, I, 7 ; Diodore, XXIII, $1,4$.

(59) Diodorf, XXII, 2, 2. G. Manganaro, "Metoikismos - Metaphora di poleis in Sicilia: il caso di Geloi di Phintias e la relativa documentazione épigrafica», ANSP, 1990, pp. 391-408. 
instable qu'est la Sicile. Tout tyran cependant ne devient pas fondateur : il y faut des conditions favorables ordinaires, comme la situation géographique de la cité du tyran, ou conjoncturelles, comme la destruction d'une cité par l'ennemi ou la nature. La politique de Gélôn d'une grande Syracuse a servi de modèle pendant deux siècles, au point que Camariniens et Léontins devaient avoir l'impression d'un horrible bégaiement de l'histoire. La Sicile des tyrans est le pays de l'ảioıkía continue, le pays de l'instabilité, où le lien entre communauté humaine et territoire est sans cesse menacé par les projets de dynastes qui se veulent maîtres de l'espace et du temps.

Anne JACQuemin (Strasbourg) 